Received: 12 January 2017

Accepted: 29 August 2017

Published online: 11 September 2017

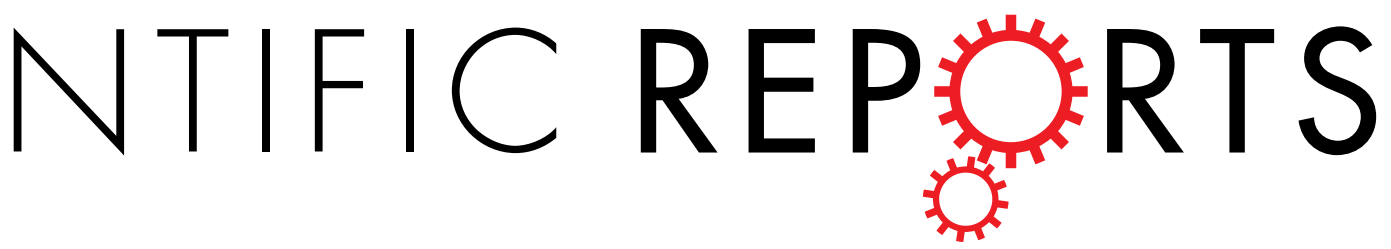

\title{
OPEN A signal-on built in-marker electrochemical aptasensor for human prostate-specific antigen based on a hairbrush-like gold
} nanostructure

\author{
Naghmeh Sattarahmady ${ }^{1,2}$, Amid Rahi ${ }^{3}$ \& Hossein Heli ${ }^{1}$ \\ A green electrodeposition method was firstly employed for the synthesis of round hairbrush-like gold \\ nanostructure in the presence of cadaverine as a size and shape directing additive. The nanostructure \\ which comprised of arrays of nanospindles was then applied as a transducer to fabricate a signal-on \\ built in-marker electrochemical aptasensor for the detection of human prostate-specific antigen (PSA). \\ The aptasensor detected PSA with a linear concentration range of 0.125 to $128 \mathrm{ng} \mathrm{mL}^{-1}$ and a limit of \\ detection of $50 \mathrm{pg} \mathrm{mL}^{-1}$. The aptasensor was then successfully applied to detect PSA in the blood serum \\ samples of healthy and patient persons.
}

Prostate cancer (PCa) is the most common cancer in men and is the second-leading cause of cancer mortality in men. This cancer accounts for sixth percent of the total cancer deaths in males ${ }^{1}$. Therefore, early diagnosis is very important to prevent PCa.

Prostate-specific antigen (PSA) is a biomarker that is most widely employed for the detection of $\mathrm{PCa}^{2}$. It has been shown that PSA is the most validated biomarker for the early detection of prostate cancer and monitoring the disease recurrence after treatment ${ }^{3}$. PSA is a glycoprotein with chymotrypsin-like protease activity and exists naturally in the human serum, either free or in combination with various proteinase inhibitors ${ }^{4}$. Increment in the PSA concentration to $>4.0 \mathrm{ng} \mathrm{mL}^{-1}$ is usually suspected to the appearance of tumors in the prostate and a biopsy should be performed ${ }^{5}$. A total PSA level higher than $10 \mathrm{ng} \mathrm{mL}^{-1}$ is generally considered with $\mathrm{PCa}^{6}$. Therefore, the development of reliable, specific, rapid and simple methods for determination of PSA is very important in the early diagnosis of $\mathrm{PCa}$ or monitoring of the disease after treatment.

Up to now, different methods have been reported for the detection of PSA including radioimmunoassay ${ }^{7}$, fluorescence detection ${ }^{8}$, enzyme-linked immunosorbent assay ${ }^{9}$, surface plasmon resonance ${ }^{10}$, chemiluminescent immunoassay ${ }^{11}$, surface-enhanced Raman scattering ${ }^{12}$, and electrochemical methods ${ }^{13}$. Some of these methods employ antibody assays which are expensive, inactive, inconvenient, time-consuming and complicated, and suffer from the disadvantages of antibodies related to the production, stability, and manipulation ${ }^{14,15}$. In addition, biosensors based on PSA aptamers with optical ${ }^{16,17}$ or electrochemical ${ }^{5,18-20}$ transductions have been reported. Aptamers, as artificial single-stranded DNA or RNA, selectively bind to small biomolecules and complex species such as cells ${ }^{21}$. Aptamers are one of the suitable choices for recognition due to their advantages including high binding affinity, good stability, high specificity, and wide range of detection ${ }^{22}$. However, already reported electrochemical PSA aptasensors suffered from the drawbacks of complexity of fabrication ${ }^{5,19}$, multiple materials and binding steps needed for immobilization of aptamers ${ }^{5,18}$, or high cost of the detection devices ${ }^{20}$. Therefore, development of novel biosensors for PSA detection is highly interested. Besides, electrochemical methods have advantages. Some of them are ease of miniaturization, high sensitivity, low cost, and rapid response ${ }^{23,24}$.

Nanotechnology plays a principal role in the fabrication of electrochemical sensors and biosensors ${ }^{25-31}$. Modification of the electrode surface by novel nanomaterials is an effective step to improve the performance of

${ }^{1}$ Nanomedicine and Nanobiology Research Center, Shiraz University of Medical Sciences, Shiraz, Iran. ${ }^{2}$ Department of Medical Physics, School of Medicine, Shiraz University of Medical Sciences, Shiraz, Iran. ${ }^{3}$ Student Research Committee, School of Medicine, Shahid Beheshti University of Medical Sciences, Tehran, Iran. Correspondence and requests for materials should be addressed to H.H. (email: heli@sums.ac.ir) 


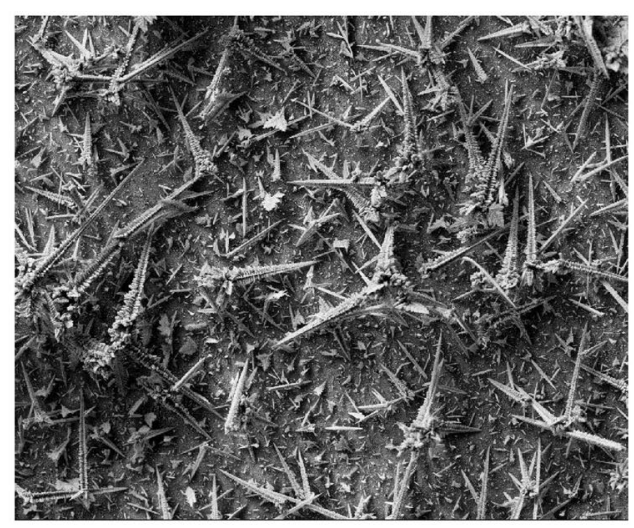

$\stackrel{10 \mu \mathrm{m}}{\longmapsto}$

(A)

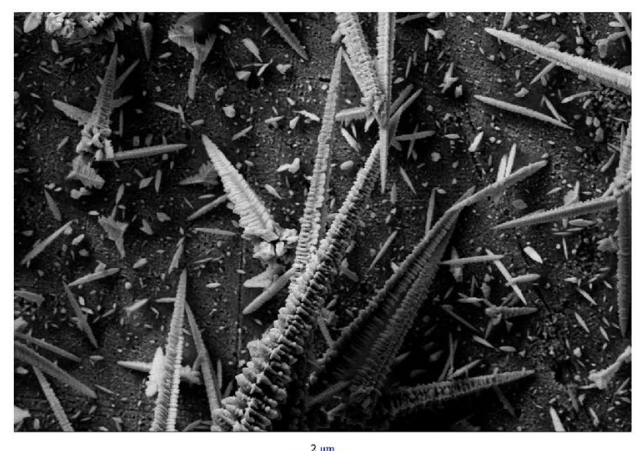

(B)

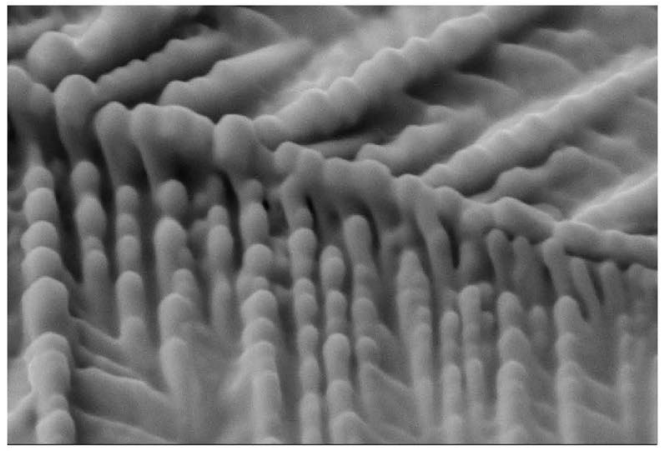

$\stackrel{200 \mathrm{~nm}}{\longmapsto}$

(C)

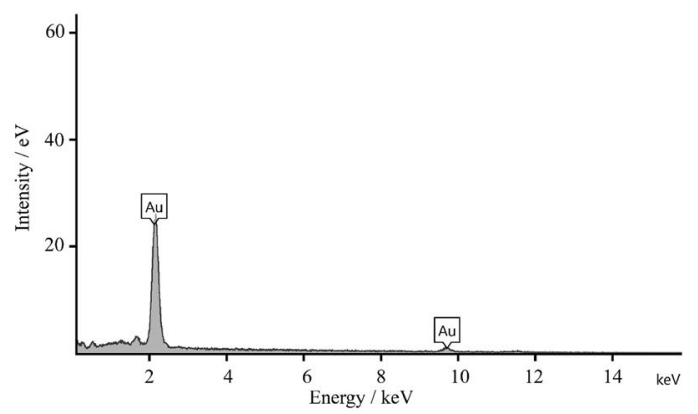

(D)

Figure 1. FESEM images with different magnifications (A-C) and an EDS spectrum (D) of the HL-Au electrode surface.

electrochemical aptasensors ${ }^{32}$. Due to the advantages of gold nanostructures including high surface area and electron transfer rate, and unique chemical characteristics, they are promising nanomaterials for immobilization of aptamers $^{33,34}$. In addition, gold nanostructures are being used to improve the selectivity and sensitivity of detection $^{28,33-37}$. A combination of aptamers with nanomaterials to fabricate electrochemical PSA biosensors provide sensitive and specific methods of PSA determination ${ }^{5,34,38}$. In the present study a built in-marker aptamer was employed for PSA detection based on a transducer of hairbrush-like gold nanostructure.

Figure 1 presents FESEM images (A-C) of the HL-Au electrode surface with different magnifications. Low-magnified images indicate that the surface was covered by round hairbrush-like rods. High-magnified image indicates that the rods surface comprised of spindles of $30-150 \mathrm{~nm}$ in diameter. Figure $1 \mathrm{D}$ shows an EDS spectrum of the hairbrush-like gold nanostructure confirming that the deposited layer comprised of pure gold. The shape and size of the hairbrush-like gold nanostructure provides an appropriate substrate (as a transduction element) for aptamer immobilization, and can promote aptamer-PSA binding ${ }^{39}$. Real surface area of the hairbrush-like gold nanostructure was measured, and it was obtained that the surface area of the HL-Au electrode is $\sim 1.6$ times of the Au electrode. Therefore, the HL-Au electrode has a larger surface area providing a high surface concentration of immobilized aptamer for a higher sensitive biosensing.

For electrodeposition of the hairbrush-like gold structure a negative overpotential of $0 \mathrm{~V}$ (vs. $\mathrm{AgCl}$ ) was applied. This leads to a fast gold atom formation ${ }^{40}$ and producing many randomly distributed nuclei at the surface. On the other hand, cadaverine is simultaneously adsorbed on the $\left(\begin{array}{lll}1 & 1 & 1\end{array}\right)$ plane of the nuclei ${ }^{41}$. This is due to the binding affinity of polyamines (such as cadaverine) to the gold surface ${ }^{42-44}$. Cadaverine also provides a positive charge surface due to its protonation in the synthesis solution, and therefore, more adsorption of $\mathrm{AuCl}_{4}{ }^{-}$ions on the ( $\left.\begin{array}{lll}1 & 1 & 1\end{array}\right)$ plane occurs following via preferential growth along the (lll 111$)$ directions by the diffusion-limited aggregation mechanism ${ }^{45}$.

DPVs recorded for the aptasensor in Tris before and after binding with different concentrations of PSA are shown in Fig. 2A. PSA binding induces aptamer folding into a new structure decreasing the distance between MB and the aptasensor surface. Therefore, the electron transfer efficiency is accelerated. Figure $2 \mathrm{~B}$ shows the dependency of the DPVs peak currents on the PSA concentration. This calibration plot is linear in the range of 0.125 to $128 \mathrm{ng} \mathrm{mL}^{-1}$ of PSA with a regression equation of $\mathrm{y}=-(0.032 \pm 0.0006) \mathrm{x}+(0.0562 \pm 0.0007)$. Based on this plot, a limit of detection (LOD, 38/m) of $0.04 \mathrm{ng} \mathrm{mL}^{-1}$ for PSA was attained. In Table 1, a comparison between LOD values reported for the PSA detection methods is summarized.

In order to inspection the selectivity of the aptasensor, DPVs for binding the non-specific proteins of hemoglobin and bovine serum albumin were recorded and shown in Fig. 3. The peak currents had a little decrement. 

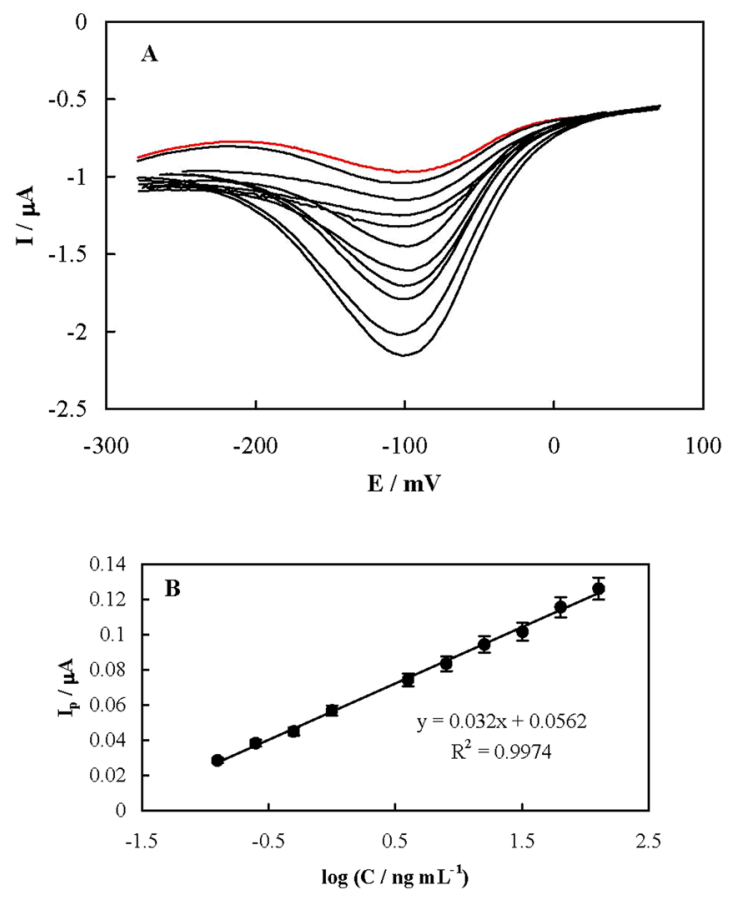

Figure 2. (A) DPVs recorded using the aptasensor in Tris before (red line) and after (black lines) binding with different concentrations of PSA of $0.125,0.25,0.5,1.0,2.0,4.0,8.0,16,32,64$ and $128 \mathrm{ng} \mathrm{mL}{ }^{-1}$. The arrow shows the direction of PSA concentration increment. (B) The dependency of the peak current on the PSA concentration (the calibration curve).

It should also be noticed that the blood serum carries different biologicals such as enzymes, proteins, hormones and antibodies with high concentrations. The aptasensor could directly analyze PSA in the serum samples with small values of constant error, and therefore, it can be considered as selective.

In order to apply the aptasensor for PSA detection in real samples, blood serum samples of healthy and patient persons (already confirmed by an immunoradiometric assay) were analyzed. It was obtained that peak currents in DPVs recorded for the healthy samples were near the same as that for the absence of PSA (data not shown). The PSA levels of the patient samples could be quantified, and compared with those obtained by the immunoradiometric assay in Table 2. The results indicated that the aptasensor can be applied for PSA detection in patients and clinical diagnosis.

Reproducibility of fabrication of the aptasensor was inspected by immersion in the Piranha solution for $30 \mathrm{~s}$ and re-fabrication $(\mathrm{n}=4)$, and DPVs were recorded (Fig. 4). The results showed a relative standard deviation (RSD) of $3.8 \%$ for the peak current.

In order to inspect the regeneration of the aptasensor, it bonded with PSA for $1 \mathrm{~h}$ at $37^{\circ} \mathrm{C}$ and then immersed in hot water of $70^{\circ} \mathrm{C}$ for $5 \mathrm{~min}$ to release PSA. Then it was cooled down slowly to room temperature and re-bonded with the same PSA concentration $(\mathrm{n}=4)$. DPVs were recorded after repeated binding with $1.0 \mathrm{ng} \mathrm{mL} \mathrm{PSA}^{-1}$ and shown in Fig. 5. Based on the data, a RSD of $4.1 \%$ was obtained for regeneration of the aptasensor.

To evaluate the stability of the aptasensor, DPVs were recorded for $1.0 \mathrm{ng} \mathrm{mL}^{-1}$ PSA in consecutive days, and aptasensor was stored in Tris at $4^{\circ} \mathrm{C}$. The peak current did not regularly change at least for 25 days.

In summary, hairbrush-like gold nanostructure was firstly electrodeposited in the presence of cadaverine. It seems that the molecules bearing multiple amine functional groups can enforce gold nucleation and growth process in a special direction, and produce special shape and size of gold nanostructures. A specific aptamer for PSA was then modified with MB (as a marker) to produce a built in-marker aptamer. The gold nanostructure, as a transducer, was employed for immobilization of a high surface concentration of the aptamer and fabrication of an aptasensor. Aptasensor was signal-on type, had a low LOD for PSA, can detect PSA in patients' samples, and would be applicable for clinical analysis.

\section{Methods}

Materials. All chemicals were of analytical grade from Scharlau (Spain) or Merck (Germany). All solutions were prepared by redistilled water. A specific aptamer sequence for PSA which was modified with methylene blue $(\mathrm{MB})$ was employed with following sequence. It has been reported that this sequence has a high affinity to PSA $^{46}$ and modified with MB in this study: $5^{\prime} \mathrm{SH}-\left(\mathrm{CH}_{2}\right)_{6}$ TT TT TA AT TA AA GC TC GC CA TC AA AT AG CT TT-3'-MB.

The aptamer was purchased from Bioneer (Korea). PSA, hemoglobin and bovine serum albumin were purchased form Sigma (USA). The aptamer stock solutions were prepared with a $20 \mathrm{mmol} \mathrm{dm}^{-3}$ Tris- $\mathrm{HCl}$ buffer, $\mathrm{pH}$ 7.4 solution (Tris) and kept frozen. 


\begin{tabular}{|c|c|c|c|c|}
\hline Detection method & Recognition element & Linear range $\left(\mathrm{ng} \mathrm{mL}^{-1}\right)$ & LOD $\left(p g \mathrm{~mL}^{-1}\right)$ & Reference \\
\hline Fluorescence & Dye-labeled aptamer/MoS ${ }_{2}$ nanosheets & $0.5-300$ & 200 & 16 \\
\hline Chemiluminescence & $\begin{array}{l}\text { Dye-labeled aptamer } / \mathrm{Fe}_{3} \mathrm{O}_{4} \text {-graphene oxide } \\
\text { nanoparticles }\end{array}$ & $1.6-50$ & 500 & 17 \\
\hline Electrochemistry & Aminated aptamer & $1-100$ & 1000 & 18 \\
\hline Electrochemistry & Biotinated aptamer & $0.25-200$ & 250 & 5 \\
\hline Electrochemistry & Aptamer-MIP hybrid & $0.1-100$ & 1 & 19 \\
\hline Electrochemical impedance & Aptamer & $0.007-6$ & 1000 & 20 \\
\hline Electrochemistry & Aptamer & $0.125-200$ & 50 & 34 \\
\hline Surface acoustic wave detection & Aptamer beacon & $10-1000$ & $1.0 \times 10^{4}$ & 50 \\
\hline RLS assay & Aptamer-modified AuNPs & $0.13-110$ & 320 & 51 \\
\hline Electrochemical immunoassay & Label-free PSA antigen & $0.05-5$ & 13 & 52 \\
\hline Electrochemical immunoassay & Graphene/cobalt hexacyanoferrate & $0.02-2$ & 100 & 53 \\
\hline Electrochemical immunoassay & Magnetic beads enzyme linked immunosorbent & $0-1$ & $<100$ & 54 \\
\hline Voltammetric ELISA & DAB- $\mathrm{H}_{2} \mathrm{O}_{2}-\mathrm{HRP}$ & $0.2-32$ & 100 & 55 \\
\hline Electrochemical immunoassay & $A b_{1}-A g-A b_{2}-H R P$ & $0-15$ & 250 & 56 \\
\hline Electrochemical dual sensing & Enzyme/antibody & $1-10$ & 1000 & 57 \\
\hline Colorimetric assay & PSA peptide & $0.1-100$ & $1.0 \times 10^{4}$ & 58 \\
\hline Optical & Antibody & $0.0005-5$ & 10 & 59 \\
\hline Duplexed immunoassay & Functional microbeads & $0.01-5$ & 50 & 60 \\
\hline Cathodic ECL immunoassay & Antibody/luminal/graphene & $0.01-8$ & 8 & 61 \\
\hline Sandwich-type immunoassay & Antibody on PDMS microfluidic chips & $4-10$ & 520 & 62 \\
\hline Chemiluminescence & Dye-labeled aptamer & $1.9-125$ & $1.0 \times 10^{3}$ & 63 \\
\hline SIA & Antibody & $0.001-1000$ & 0.11 & 64 \\
\hline ECL & Sandwich-type immunoreaction & $0.001-10$ & 0.3 & 65 \\
\hline Electrochemistry & Graphene oxide/ssDNA/PLLA nanoparticles & $0.05-100$ & $1.0 \times 10^{3}$ & 66 \\
\hline Bridge-shaped PZT resonator & Antibody & $0.01-0.1$ & $4.0 \times 10^{3}$ & 67 \\
\hline Magnetic immunoassay & SERS-based microdroplet & 174 droplets per minute & 100 & 68 \\
\hline Electrochemistry & Peptide cleavage & $0.001-30$ & 0.78 & 69 \\
\hline Imprinted capacitive biosensor & Microcontact-PSA-MIP & $0.00001-100$ & 0.6 & 70 \\
\hline Microarray immunoassay & Antibody & $0.005-500$ & 5 & 71 \\
\hline Electrochemistry & Aptamer-attached marker (MB) & $0.125-128$ & 40 & This work \\
\hline
\end{tabular}

Table 1. A comparison between the PSA detection methods. Abbreviations: $A b_{1}-A g-A b_{2}-H R P$ :

Antibody1-antigene-antibody 2- horseradish peroxidase. AuNPs: Gold nanoparticles. DAB- $\mathrm{H}_{2} \mathrm{O}_{2}-\mathrm{HRP}$ : 3,3'-diaminobenzidine- $\mathrm{H}_{2} \mathrm{O}_{2}$-horseradish peroxidase. ECL: Electrochemiluminescence. LOD: Limit of detection. MB: Methylene blue. MIP: Molecularly imprinted polymer. PDMS: Poly(dimethylsiloxane). PLLA: Poly-L-lactide. PZT: Lead zirconate titanate. RLS: Resonance light scattering. SIA: Surface-enhanced Raman scattering (SERS)-based immunoassays. ssDNA: Single stranded DNA.

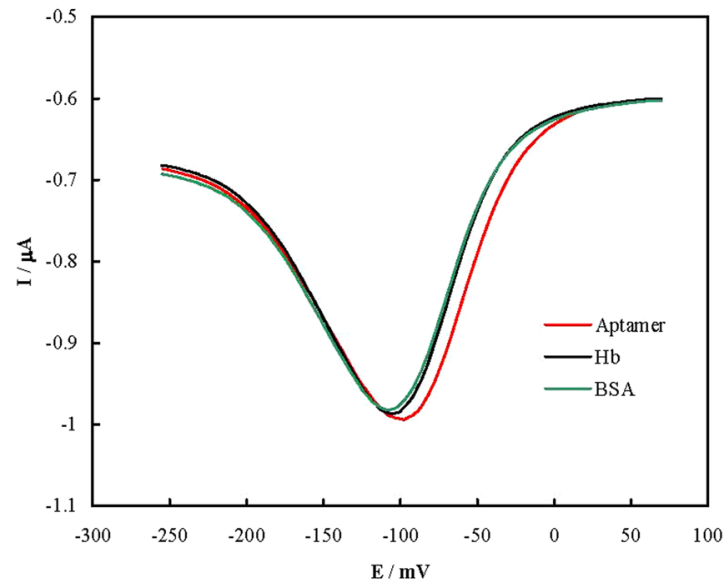

Figure 3. DPVs recorded using the aptasensor in Tris before and after binding with $50 \mathrm{mg} \mathrm{mL}^{-1}$ bovine serum albumin (green) or $17.5 \mathrm{mg} \mathrm{mL}^{-1}$ hemoglobin (black). 


\begin{tabular}{|c|c|c|}
\hline Nominal value $^{\mathrm{a}}\left(\mathrm{ng} \mathrm{mL}^{-1}\right)$ & Obtained value $\left(\mathrm{ng} \mathrm{mL}^{-1}\right)$ & Bias (\%) \\
\hline 5.31 & 5.2 & -2.1 \\
\hline 6.78 & 6.94 & 2.4 \\
\hline 6.87 & 6.99 & 1.7 \\
\hline 8.48 & 8.54 & 0.7 \\
\hline 8.71 & 8.72 & 0.1 \\
\hline 9.19 & 8.95 & -2.6 \\
\hline 10.92 & 11.01 & 0.8 \\
\hline 12.31 & 11.88 & -3.5 \\
\hline 13.31 & 13.60 & 2.2 \\
\hline 19.00 & 18.46 & -2.8 \\
\hline
\end{tabular}

Table 2. Values of the PSA concentrations in the patients' blood serum obtained by the aptasensor. ${ }^{\text {anominal }}$ values were obtained by the immunoradiometric assay.

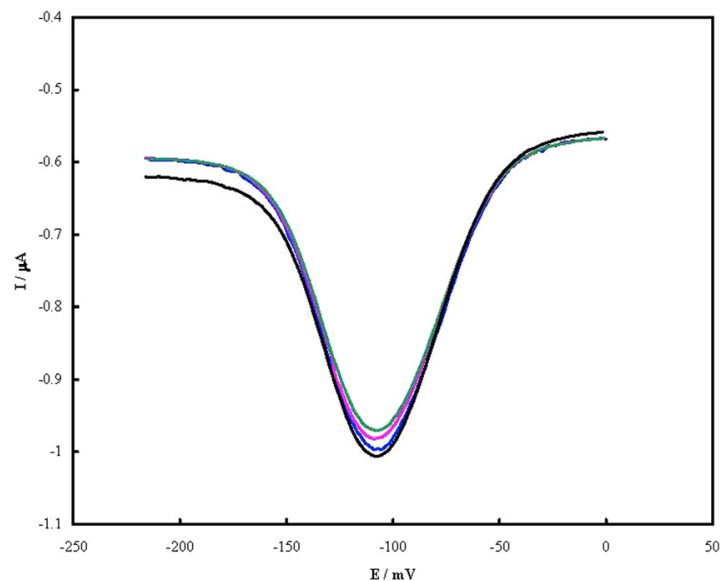

Figure 4. DPVs of the aptasensor in Tris before for the repeating fabrication.

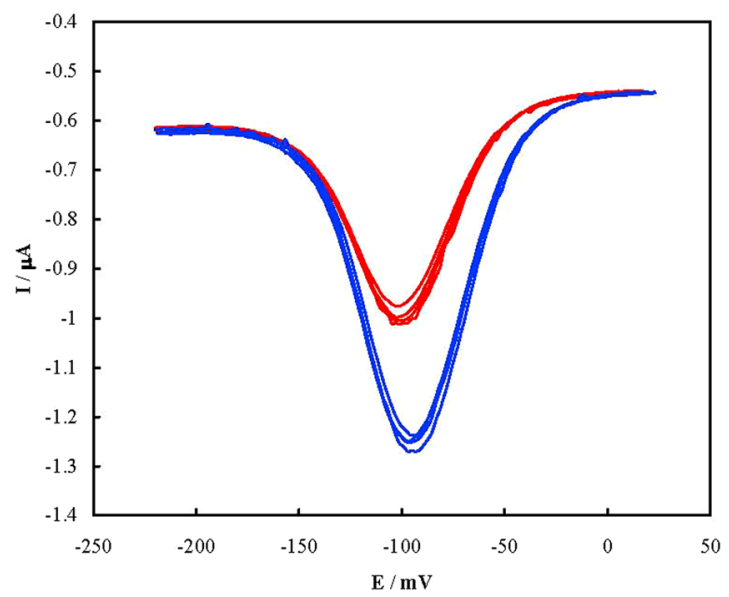

Figure 5. DPVs of the aptasensor in Tris before (red curves) and after (blue curves) reparative binding with $1.0 \mathrm{ng} \mathrm{mL}^{-1}$ PSA.

Apparatus. Electrochemical measurements were performed in a three-electrode cell connected to a $\mu$-Autolab potentiostat/galvanostat (the Netherlands). $\mathrm{An} \mathrm{Ag} / \mathrm{AgCl}, 3 \mathrm{~mol} \mathrm{dm}{ }^{-3} \mathrm{KCl}$, a platinum rod, and a gold disk ( $\mathrm{Au}, 2 \mathrm{~mm}$ of diameter) or the Au electrode deposited with hairbrush-like gold nanostructure (HL-Au electrode) were employed as the reference, counter and working electrodes, respectively. The system was run on a PC by GPES 4.9 software. 
Field emission scanning electron microscopy (FESEM) was performed using a Zeiss, Sigma-IGMA/VP (Germany) equipped with energy-dispersive X-ray spectroscopy (EDS). The samples were coated by a $2-5 \mathrm{~nm}$ thin film of gold by sputtering.

Preparation of HL-Au electrode. Firstly, the Au electrode was polished on a polishing pad with 0.05 $\mu \mathrm{m}$-alumina powder lubricated by water. Polishing was continued to attain a mirror-like surface. The electrode was then cleaned by immersion in a 1:3 water/ethanol mixture and ultrasonication for $8 \mathrm{~min}$ in an ultrasound bath. It was further electropolished in a $500 \mathrm{mmol} \mathrm{dm}{ }^{-3} \mathrm{H}_{2} \mathrm{SO}_{4}$ solution and applying cyclic potential in the range of cathodic to anodic edges of the electrolyte stability for 20 consecutive cycles. The Au electrode was then transferred to a cell containing the electrodeposition solutions containing $20 \mathrm{mmol} \mathrm{dm}^{-3} \mathrm{HAuCl}_{4}+500 \mathrm{mmol} \mathrm{dm}^{-3}$ $\mathrm{H}_{2} \mathrm{SO}_{4}+150 \mathrm{mmol} \mathrm{dm}^{-3}$ cadaverine. Electrodeposition was done at $0 \mathrm{mV}$ for $600 \mathrm{~s}$. The nsAu electrode was then rinsed thoroughly with distilled water.

Immobilization of the aptamer. Lyophilized aptamer was dissolved in distilled water. Then, $10 \mu \mathrm{L}$ dithiothreitol (DTT) solution (containing $10 \mathrm{mmol} \mathrm{dm}^{-3}$ sodium acetate, $\mathrm{pH} 5.2$ and $500 \mathrm{mmol} \mathrm{dm}^{-3} \mathrm{DTT}$ ) was added, mixed, and incubated at room temperature for $15 \mathrm{~min}$. Excess DTT and thiol fragments were removed from the mixture by extraction with ethyl acetate (three times, total volume of $150 \mu \mathrm{L}$ ), and the upper layer was discarded, the next step was then immediately performed. Immobilization of aptamer was done by dropping $10 \mu \mathrm{L}$ of 10.0 $\mu \mathrm{mol} \mathrm{dm} \mathrm{d}^{-3}$ aptamer solution dissolved in Tris on the HL-Au electrode surface and kept at $4{ }^{\circ} \mathrm{C}$ for $8 \mathrm{~h}^{11,28,34}$. Then, the electrode was rinsed with Tris, and further treated with $1.0 \mathrm{mmol} \mathrm{dm}^{-3} 6$-mercapto-1-hexanol at room temperature for $30 \mathrm{~min}$ to obtain a well aligned aptamer monolayer ${ }^{11,28,34}$. Then, the electrode was washed again with Tris and double distilled water, respectively, to remove non-specific adsorbed thiols. The obtained electrode was denoted as aptasensor

PSA binding. PSA binding process was performed by immersing the aptasensor into Tris containing various concentrations of PSA for $1 \mathrm{~h}$ at $37^{\circ} \mathrm{C}^{47,48}$ and then rinsed with Tris.

Electrochemical measurements. The real surface areas of the Au and HL-Au electrodes were electrochemically determined. The electrodes were transferred to a solution of $0.5 \mathrm{~mol} \mathrm{dm}^{-3} \mathrm{KCl}$ containing $0.5 \mathrm{mmol} \mathrm{dm}^{-3} \mathrm{~K}_{4}\left[\mathrm{Fe}(\mathrm{CN})_{6}\right]$, and cyclic voltammograms at different potential sweep rates were recorded. Using the Randles-Sevcik equation ${ }^{25}$ and the value of $7.60 \times 10^{-6} \mathrm{~cm} \mathrm{~s}^{-1}$ for the diffusion coefficient of $\left[\mathrm{Fe}(\mathrm{CN})_{6}\right]^{4-49}$, the real surface areas of the Au and HL-Au electrodes were measured.

Electrochemical detection of PSA was done in an electrochemical cell containing $10 \mathrm{~mL}$ Tris by recording differential pulse voltammograms (DPVs) for the reduction peak of aptamer built in-MB. DPVs were recorded with a pulse width of $25 \mathrm{mV}$, a pulse time of $50 \mathrm{~ms}$, and a scan rate of $10 \mathrm{mV} \mathrm{s}^{-1}$. All electrochemical measurements were performed at room temperature.

Human samples. Blood samples of patient and healthy persons in our study provided by Faghihi Hospital Shiraz (Iran), and approved by Ethics Committee of Shiraz University of Medical Sciences (Number 12292). All methods were performed in accordance with the approved guidelines, and the institutional review board waived the need to informed consent for patient samples. The samples were taken by laboratory of the hospital and were kept until certain time. All collected samples were kept frozen until assay. The PSA levels of the samples were firstly determined by an immunoradiometric assay, and then, were diluted with distilled water (1:9) and assayed by aptasensor.

\section{References}

1. Welch, H. G. et al. Trends in metastatic breast and prostate cancer. N. Engl. J. Med. 374, 594-596 (2016).

2. Ankerst, D. P. et al. Serial percent-free PSA in combination with PSA for population-based early detection of prostate cancer. J. Urol. 196, 355-360 (2016).

3. Carter, H. B. et al. Early detection of prostate cancer: AUA guideline. J. Urol. 190, 419-426 (2013).

4. Prcic, A., Begic, E. \& Hiros, M. Usefulness of total PSA value in prostate diseases diagnosis. Acta Inform. Med. 24, 156-161 (2016).

5. Liu, B., Lu, L., Hua, E., Jiang, S. \& Xie, G. Detection of the human prostate-specific antigen using an aptasensor with gold nanoparticles encapsulated by graphitized mesoporous carbon. Microchim. Acta 178, 163-170 (2012).

6. Tosoian, J. J. et al. Prediction of pathological stage based on clinical stage, serum prostate-specific antigen, and biopsy Gleason score: partin tables in the contemporary era. BJU Int. 29, 13575 (2016).

7. Graves, H., Wehner, N. \& Stamey, T. A. Ultrasensitive radioimmunoassay of prostate-specific antigen. Clin. Chem. 38, 735-742 (1992).

8. Harma, H. et al. Zeptomole detection sensitivity of prostate-specific antigen in a rapid microtitre plate assay using time-resolved fluorescence. Luminescence 15, 351-355 (2000).

9. Liang, J. et al. Silver nanoprism etching-based plasmonic ELISA for the high sensitive detection of prostate-specific antigen. Biosens. Bioelectron. 69, 128-134 (2015).

10. Breault-Turcot, J., Poirier-Richard, H. P., Couture, M., Pelechacz, D. \& Masson, J. F. Single chip SPR and fluorescent ELISA assay of prostate specific antigen. Lab Chip 15, 4433-4440 (2015).

11. Liu, A., Zhao, F., Zhao, Y., Shangguan, L. \& Liu, S. A portable chemiluminescence imaging immunoassay for simultaneous detection of different isoforms of prostate specific antigen in serum. Biosens. Bioelectron. 81, 97-102 (2016).

12. Yeh, S. H. et al. Wash-free magnetic immunoassay of the PSA cancer marker using SERS spectroscopy and droplet microfluidics. $L a b$ Chip 16, 622-622 (2016).

13. Chuah, K. et al. Ultrasensitive electrochemical detection of prostate-specific antigen (PSA) using gold-coated magnetic nanoparticles as 'dispersible electrodes'. Chem. Commun. 48, 3503-3505 (2012).

14. Lee, J. H., Rho, J. E. R., Rho, T. H. D. \& Newby, J. G. Advent of innovative chemiluminescent enzyme immunoassay. Biosens. Bioelectron. 26, 377-382 (2010).

15. Panini, N. V., Messina, G. A., Salinas, E., Fernandez, H. \& Raba, J. Integrated microfluidic systems with an immunosensor modified with carbon nanotubes for detection of prostate specific antigen (PSA) in human serum samples. Biosens. Bioelectron. 23, 1145-1151 (2008). 
16. Mei, K. R., Ding, L., Wang, Z., You, J. \& Qu, F. A novel aptamer-functionalized MoS2 nanosheet fluorescent biosensor for sensitive detection of prostate specific antigen. Anal. Bioanal. Chem. 2, 369-377 (2015).

17. Kyu, C. H. \& Lee, J. H. Role of magnetic Fe3O4 graphene oxide in chemiluminescent aptasensors capable of sensing tumor markers in human serum. Anal. Method 24, 6964-6968 (2013).

18. Souada, M. et al. Label-free electrochemical detection of prostate-specific antigen based on nucleic acid aptamer. Biosens. Bioelectron. 68, 49-54 (2015)

19. Pawan, J. et al. Aptamer-MIP hybrid receptor for highly sensitive electrochemical detection of prostate specific antigen. Biosens. Bioelectron. 75, 188-195 (2016).

20. Pawan, J. et al. Label-free impedimetric aptasensor with antifouling surface chemistry: A prostate specific antigen case study. Sens. Actuators B: Chem. 209, 306-312 (2015).

21. Chandola, C., Kalme, S., Casteleijn, M. G., Urtti, A. \& Neerathilingam, M. Application of aptamers in diagnostics, drug-delivery and imaging. J. Biosci. 41, 535-561 (2016).

22. Song, S., Wang, L., Li, J., Fan, C. \& Zhao, J. Aptamer-based biosensors. Trends Anal. Chem. 27, 108-117 (2008).

23. Bard, A. J. \& Faulkner, L. R. Electrochemical Methods, 2nd ed., (John Wiley and Sons, New York, 2001).

24. Rahi, A., Karimian, K. \& Heli, H. Nanostructured materials in electroanalysis of pharmaceuticals. Anal. Biochem. 497, 39-47 (2016).

25. Vais, R. D., Sattarahmady, N., Karimian, K. \& Heli, H. Green electrodeposition of gold hierarchical dendrites of pyramidal nanoparticles and determination of azathioprine. Sens. Actuators B Chem. 215, 113-118 (2015).

26. Rahi, A., Sattarahmady, N., Vais, R. D. \& Heli, H. Sonoelectrodeposition of gold nanorods at a gold surface- Applicationfor electrocatalytic reduction and determination of nitrofurazone. Sens. Actuators B Chem. 210, 96-102 (2015).

27. Sattarahmady, N., Heli, H. \& Vais, R. D. An electrochemical acetylcholine sensor based on lichen-like nickel oxide nanostructure. Biosens. Bioelectron. 48, 197-202 (2013).

28. Rahi, A., Sattarahmady, N. \& Heli, H. Zepto-molar electrochemical detection of Brucella genome based on gold nanoribbons covered by gold nanoblooms. Sci. Rep. 5, 18060 (2015).

29. Heli, H., Pishahang, J. \& Barzegar Amiri, H. Synthesis of hexagonal CoAl-layered double hydroxide nanoshales/carbon nanotubes composite for the non-enzymatic detection of hydrogen peroxide. J. Electroanal. Chem. 768, 134-144 (2016).

30. Sattarahmady, N., Heli, H. \& Moradi, S. E. Cobalt hexacyanoferrate/graphene nanocomposite-Application for the electrocatalytic oxidation and amperometric determination of captopril. Sens. Actuators B Chem. 177, 1098-1106 (2013).

31. Rahi, A., Sattarahmady, N. \& Heli, H. An ultrasensitive electrochemical genosensor for Brucella based on palladium nanoparticles. Anal. Biochem. 510, 11-17 (2016).

32. Palchetti, I. \& Mascini, M. Electrochemical nanomaterial-based nucleic acid aptasensors. Anal. Bioanal. Chem. 402, 3103-3114 (2012).

33. Wen, W. et al. Increased electrocatalyzed performance through hairpin oligonucleotide aptamer-functionalized gold nanorods labels and graphene-streptavidin nanomatrix: Highly selective and sensitive electrochemical biosensor of carcinoembryonic antigen. Biosens. Bioelectron. 83, 142-148 (2016).

34. Rahi, A., Sattarahmady, N. \& Heli, H. Label-free electrochemical aptasensing of the human prostate-specific antigen using gold nanospears. Talanta 156-157, 218-224 (2016).

35. Moradi, M. et al. A label-free, PCR-free and signal-on electrochemical DNA biosensor for Leishmania major based on gold nanoleaves. Talanta 161, 48-53 (2016).

36. Sattarahmady, N., Kayani, Z. \& Heli, H. Highly simple and visual colorimetric detection of Brucella melitensis genomic DNA in clinical samples based on gold nanoparticles. J. Iran. Chem. Soc. 12, 1569-1576 (2015).

37. Moradi, M., Sattarahmady, N., Hatam, G. R. \& Heli, H. Electrochemical genosensing of Leishmania major using gold hierarchical nanoleaflets. J. Biol. Today's World 5, 128-136 (2016).

38. Zhu, Y., Wang, H., Wang, L., Zhu, J. \& Jiang, W. Cascade signal amplification based on copper nanoparticle-reported rolling circle amplification for ultrasensitive electrochemical detection of the prostate cancer biomarker. ACS Appl. Mater. Interfaces 8, 2573-2581 (2016).

39. Soleymani, L., Fang, Z., Sargent, E. H. \& Kelley, S. O. Programming the detection limits of biosensors through controlled nanostructuring. Nat. Nanotechnol. 4, 844-848 (2009).

40. Wang, J. et al. An invisible template method toward gold regular arrays of nanoflowers by electrodeposition. Langmuir 29, 3512-3517 (2013).

41. Wright, L. B., Merrill, N. A., Knecht, M. R. \& Walsh, T. R. Structure of arginine overlayers at the aqueous gold interface: Implications for nanoparticle assembly. ACS Appl. Mater. Interfaces 13, 10524-10533 (2014).

42. Fernandez-Lodeiro, A. et al. Polyamine ligand-mediated self-assembly of gold and silver nanoparticles into chainlike structures in aqueous solution: Towards new nanostructured chemosensors. ChemistryOpen 2, 200-207 (2013).

43. Hata, H., Kubo, S., Kobayashi, Y. \& Mallouk, T. E. Intercalation of well-dispersed gold nanoparticles into layered oxide nanosheets through intercalation of a polyamine. J. Am. Chem. Soc. 129, 3064-3065 (2007).

44. Radecki, J., Szymanska, I., Bulgariu, L. \& Pietraszkiewicz, M. Covalent and embedment immobilization of macrocyclic polyamines on gold electrodes and their voltammetric responses towards ethene dicarboxylic acids. Electrochim. Acta 51, 2289-2297 (2006).

45. Witten, J. T. A. \& Sander, L. M. Diffusion-limited aggregation, a kinetic critical phenomenon. Phys. Rev. Let. 47, 1400 (1981).

46. Savory, N., Abe, K., Sode, K. \& kebukuro, K. Selection of DNA aptamer against prostate specific antigen using a genetic algorithm and application to sensing. Biosens. Bioelectron. 26, 1386-1391 (2010).

47. Huang, Y. et al. Electrochemical immunosensor of platelet-derived growth factor with aptamer-primed polymerase amplification. Anal. Biochem. 382, 16-22 (2008).

48. Li, L., Zhao, H., Chen, Z., Mu, X. \& Guo, L. Aptamer-based electrochemical approach to the detection of thrombin by modification of gold nanoparticles. Anal. Bioanal. Chem. 398, 563-570 (2010).

49. Wang, S. F. \& Xu, Q. Electrochemical parameters of ethamsylate at multi-walled carbon nanotube modified glassy carbon electrodes. Bioelectrochemistry 70, 296-300 (2007).

50. Feng, Z. et al. A microfluidic love-wave biosensing device for PSA detection based on an aptamer beacon probe. Sensors 6 , 13839-13850 (2015).

51. Chen, Z., Lei, Y., Chen, X., Wang, Z. \& Liu, J. An aptamer based resonance light scattering assay of prostate specific antigen. Biosens. Bioelectron. 36, 35-40 (2012).

52. Mao, K. et al. Label-free electrochemical immunosensor based on graphene/methylene blue nanocomposite. Anal. Biochem. 422, $22-27$ (2012)

53. Li, T., Yang, M. \& Li, H. Label-free electrochemical detection of cancer marker based on graphene-cobalt hexacyanoferrate nanocomposite. J. Electroanal. Chem. 655, 50-55 (2011).

54. Sarkar, P., Ghosh, D., Bhattacharyay, D., Setford, S. J. \& Turner, A. P. F. Electrochemical immunoassay for free prostate specific antigen (f-PSA) using magnetic beads. Electroanalysis 20, 1414-1420 (2008).

55. Yang, J., Bi, C., Fan, Y. \& Zhang, X. Investigation of 3, 3'-diaminobenzidine-H2O2-horseradish peroxidase voltammetric enzymelinked immunoassay system used for the detection of prostate specific antigen. Asian J. Chem. 23, 5373-5376 (2011).

56. Sarkar, P., Pal, P. S., Ghosh, D., Setford, S. J. \& Tothill, I. E. Amperometric biosensors for detection of the prostate cancer marker (PSA). Int. J. Pharma. 238, 1-9 (2002). 
57. Escamilla-Gomez, V., Hernandez-Santos, D., Gonzalez-Garcia, M. B., Pingarron-Carrazon, J. M. \& Costa-Garcia, A. Simultaneous detection of free and total prostate specific antigen on a screen-printed electrochemical dual sensor. Biosens. Bioelectron. 24, 2678-2683 (2009).

58. Ghadeer, A. Ultra-rapid colorimetric assay for protease detection using magnetic nanoparticle-based biosensors. Analyst 138, 3735-3739 (2013).

59. Zhang, T., He, Y., Wei, J. \& Que, L. Nanostructured optical microchips for cancer biomarker detection. Biosens. Bioelectron. 38, 382-388 (2012).

60. Lee, J. et al. Microarray of stimuli-responsive microbeads for duplexed immunoassay. BioChip J. 5, 158-164 (2011).

61. Xu, S., Liu, Y., Wang, T. \& Li, J. Positive potential operation of a cathodic electrogenerated chemiluminescence immunosensor based on luminol and graphene for cancer biomarker detection. Anal. Chem. 83, 3817-3823 (2011).

62. Okada, H., Hosokawa, K. \& Maeda, M. Power-free microchip immunoassay of PSA in human serum for point-of-care testing. Anal. Sci. 27, 237 (2011).

63. Timothy, C., Cho, S., Kim, Y. T. \& Lee, J. H. Rapid aptasensor capable of simply diagnosing prostate cancer. Biosens. Bioelectron. 62 , 31-37 (2014).

64. Chang, H. et al. PSA detection with femtomolar sensitivity and a broad dynamic range using SERS nanoprobes and an area-scanning method. ACS Sens. 1, 645-649 (2016).

65. Zhao, Y. et al. A CeO2-matrical enhancing ECL sensing platform based on the Bi2S3-labeled inverted quenching mechanism for PSA detection. J. Mater. Chem. B 4, 2963-2971 (2016).

66. Pan, L. H. et al. An electrochemical biosensor to simultaneously detect VEGF and PSA for early prostate cancer diagnosis based on graphene oxide/ssDNA/PLLA nanoparticles. Biosens. Bioelectron. 16, 30092-30096 (2016).

67. Hwang, D. G. et al. Label-free detection of prostate specific antigen (PSA) using a bridge-shaped PZT resonator. Microsyst. Technol. 3, 1-8 (2016)

68. Gao, R., Cheng, Z. \& Choo, J. Wash-free magnetic immunoassay of the PSA cancer marker using SERS and droplet microfluidics. Lab Chip 16, 1022-1029 (2016).

69. Xie, S., Zhang, J., Yuan, Y., Chai, Y. \& Yuan, R. An electrochemical peptide cleavage-based biosensor for prostate specific antigen detection via host-guest interaction between ferrocene and $\beta$-cyclodextrin. Chem. Commun. 51, 3387-3390 (2015).

70. Erturk, G., Hedstrom, M., Tumer, M. A., Denizli, A. \& Mattiasson, B. Real-time prostate-specific antigen detection with prostatespecific antigen imprinted capacitive biosensors. Anal. Chimica Acta 891, 120-129 (2015).

71. Lee, S. W., Hosokawa, K., Kim, S., Laurell, T. \& Maeda, M. Simple and robust antibody microarray-based immunoassay platform for sensitive and selective detection of PSA and hK2 toward accurate diagnosis of prostate cancer. Sens. Biosens. Res. 3, 105-111 (2015).

\section{Acknowledgements}

We would like to thank the Research Council of Shiraz University of Medical Sciences (12292) for supporting this research.

\section{Author Contributions}

N.S. provided the main idea of the work, A.R. performed experiments and write the manuscript draft, H.H. provided some idea and performed some data analysis. All of the authors have reviewed the final paper and insert their comments.

\section{Additional Information \\ Competing Interests: The authors declare that they have no competing interests.}

Publisher's note: Springer Nature remains neutral with regard to jurisdictional claims in published maps and institutional affiliations.

(c) (i) Open Access This article is licensed under a Creative Commons Attribution 4.0 International License, which permits use, sharing, adaptation, distribution and reproduction in any medium or format, as long as you give appropriate credit to the original author(s) and the source, provide a link to the Creative Commons license, and indicate if changes were made. The images or other third party material in this article are included in the article's Creative Commons license, unless indicated otherwise in a credit line to the material. If material is not included in the article's Creative Commons license and your intended use is not permitted by statutory regulation or exceeds the permitted use, you will need to obtain permission directly from the copyright holder. To view a copy of this license, visit http://creativecommons.org/licenses/by/4.0/.

(C) The Author(s) 2017 